\title{
Fin de vie, mort, médecine
}

\section{Jean Martin}

Dr med., membre de la rédaction

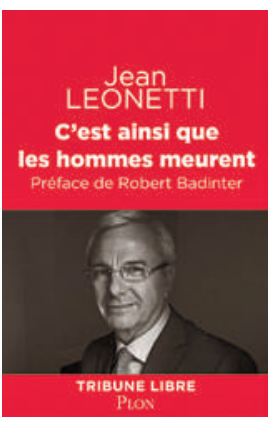

Jean Leonetti

C'est ainsi que les hommes meurent

Paris: Editions Plon/ Tribune libre; 2015. 192 pages. $23.80 \mathrm{CHF}$. ISBN

978-2-259-23010-0
Jean Leonetti (1948) est le médecin et homme politique français qui a donné son nom à la loi du 22 avril 2005 relative aux droits des malades et à la fin de vie, principal document législatif français traitant de cette problématique. A quoi toutefois il faut ajouter le rapport de décembre 2012 de la Commission de réflexion présidée par le professeur Didier Sicard - mais qui n’a pas valeur légale. On sait que le rapport Sicard décrivait comment, dit en termes bruts, "on meurt mal en France aujourd'hui». Toutefois, les observateurs relèvent que, si les dispositions de la loi Leonetti étaient appliquées, la situation serait bien plus satisfaisante; parce que, dans la pratique quotidienne et nationale, on renoncerait vraiment à l'obstination médicale déraisonnable, on traiterait mieux la douleur en abandonnant de vieilles réticences vis-à-vis des antalgiques majeurs, on prodiguerait la sédation profonde.

Le Dr Leonetti vient de publier un ouvrage sur la mort, le mourir et, en contre-chant, la vie. Panorama substantiel de ce que la réalité et l'inéluctabilité de la mort, les idées, craintes voire fantasmes qui y sont liés, ont suscité chez les philosophes, les doctrines religieuses, les écrivains. Tout en abordant les questions très actuelles posées dans la pratique et l'éthique médicales. Large réflexion marquée par la culture de l'auteur - y compris culture chrétienne.

\section{Des positions médico-éthiques avec le doigt sur la réalité}

Approcher les positions d'un confrère qui a influencé la pensée politique et médicale au cours des dernières années dans son pays m’intéressait beaucoup. Sur la «scène éthique française» en général, j’avoue certaines réserves: vis-à-vis d'une adhérence étroite à des éléments de doctrine qui valaient jusqu'ici comme intangibles, mais devraient être discutés et aménagés. Ceci en rapport avec le fait que le tableau général de la maladie et du mourir et leurs composantes socio-culturelles, et donc la pratique de la médecine, ont profondément évolué. Changements qui, couplés à la promotion des droits de patients (avec l'accent mis sur leur autonomie), dans la loi et dans la vie quotidienne, demandent des réexamens approfondis: vis-à-vis de la douleur et de sa prise en charge, de la maladie terminale, des déterminations propres des malades, y com- pris s'agissant de refus de mesures médicales (supplémentaires) et d'aide à soulager une agonie.

L'ouvrage de Jean Leonetti retient l'attention parce que, ai-je trouvé, on n'y voit pas un certain côté militant traditionnel, voire simpliste ou même rendu caricatural par des envolées rhétoriques, sur des thèmes parfois éloignés de la vie des gens, qu'on aurait imaginé chez un personnage politique: il aurait pu vouloir souligner de manière combative la "différence» ou l'«exception» française, par rapport notamment aux positions anglo-saxonnes libérales. Cela n'est pas le cas En réalité, si ce qui est dit dans ce livre était appliqué aujourd'hui chez nos voisins, la situation pratique ne serait pas aussi différente, entre eux et nous, qu'on pourrait le penser en lisant les fortes prises de position de ténors politiques et sociétaux, y compris de l'Ordre national des médecins, vouant aux gémonies toute éventualité d'admettre dans certains cas une assistance au suicide (ou pire, une euthanasie). Regrettable d'en parler de manière si manichéiste alors que, en réalité, la vie, le mourir, la médecine, la vie en société - ne sont jamais en noir pur ou blanc pur, mais sont faits de continua de nuances de gris (étant admis que, une fois la mort intervenue, on est dans une situation catégorique - noire ou blanche, chacun choisira). Et Leonetti de rappeler: «La vie, c'est changer d'avis.» Oui! Sans que je sois compétent pour en juger de manière détaillée, cela semble confirmer que, si ce que le Dr Leonetti préconise - et qui se trouve largement dans la loi qui porte son nom - était accepté, dès aujourd'hui on mourrait moins mal en France.

\section{Le mourir, la médecine, les soins palliatifs}

Quelques citations: "Ces situations d'état végétatif chronique ne sont pas le résultat d'une maladie, mais le produit d'une médecine moderne incapable de mâ̂triser sa puissance et de prévoir les résultats de son action» - obstination déraisonnable. "Les médecins génèrent quelquefois des situations de survie qui apparaissent insupportables pour les sujets qui les subissent et leur entourage. Cette remise en cause de l'action médicale déstabilise le monde des soignants, à qui il faut apprendre ou réapprendre à s'abstenir de toute action et à mieux prendre en compte la volonté 
du malade. L'adage médical 'D'abord ne pas nuire' concerne désormais des actions jugées utiles à la poursuite de la vie [biologique] mais néfastes sur le plan humain.»

"La médecine traditionnelle, bien mieux préparée à sauver et guérir qu'à accompagner et soulager, a trouvé dans la médecine palliative son complément indispensable [...] Toutefois, la médecine palliative n'intervient le plus souvent qu'après la curative, alors que les deux stratégies doivent cohabiter».

«Le médecin n'est pas le détenteur de la morale [...] La médecine n'est pas non plus l'instrument de tous les désirs et de toutes les insatisfactions. Elle doit agir non pas en fonction d'une morale médicale enfermée dans un corporatisme étroit, mais au nom de valeurs universelles de bienfaisance et de solidarité envers les plus faibles."

\section{Vie, mort et sociétés}

Au-delà des positions de Leonetti sur les enjeux d'éthique et pratique médicales, ses réflexions plus générales méritent aussi qu'on s'y arrête.

«Le problème de la mort se situe aux croisements des réflexions philosophiques, psychologiques, sociétales et religieuses. Ce n'est donc que dans un contexte de dialogue et de médiation interdisciplinaires que la fin de vie de chacun doit être abordée.»

«La façon de mourir des hommes est un marqueur du type de société dans laquelle ils vivent et interroge nos valeurs. Quelle que soit cependant la société, l'humain est partagé entre la certitude de sa finitude et le questionnement sans fin qu'elle suscite». Une réflexion quelque peu culturo-centrée: «Les raisons que l'on a de vivre sont les mêmes que celles que l'on a de mourir: Dieu, le roi, la patrie, la famille, l'humanité». A quoi, aujourd'hui on peut ajouter "soi-même»! Avec tous les accents mis sur l'auto-réalisation, sur la concrétisation souhaitée de ses propres potentiels.

Préoccupante société (hyper)médiatique: «A propos de fin de vie et de la mort, on voit comment les affaires ont souvent été abordées de manière superficielle [...] Dans l'immédiat et l'émotion, il n'est pas nécessaire d'avoir une pensée mais un avis. Cette 'société de l'opinion' va varier en fonction des sondages successifs et des questions posées [...] Ainsi les éléments sont réunis pour n'avoir de pensée sur presque rien mais une opinion sur presque tout.»

«Notre société évolue vers plus de droits et moins de devoirs. Le 'c'est mon choix' devient 'c'est mon droit'. Or ce droit à la différence peut créer une différence des droits. La vision individuelle de la mort, vécue comme 'ma mort' et non pas comme 'ma mort parmi les autres' exclut le groupe et affaiblit le deuil et les rites».

\section{Demain?}

Vie après la vie ou pas? «L'idée de la vie sans au-delà est à la fois désespérante et libératrice. S'il n'y a pas d'espoir de vie bonne après la vie terrestre, rien ne réparera les injustices du monde; mais la mort sans Dieu libère la vie en faisant peser sur les hommes la responsabilité de l'humanité - et tout leur devient désespérément possible, en particulier de décider seuls de leur vie et de leur mort.»

Prise de recul: Auparavant, "une espérance criait dans un monde de violence et de haine que chaque vie était digne et que le bonheur était possible. Aujourd'hui avec la lucidité que l'histoire nous enseigne, nous savons que Dieu restera silencieux et que le grand soir ne viendra pas. Il nous reste à construire, avec modestie et réalisme, un sens à la vie et à la mort qui soit capable de dépasser la déception de nos rêves évanouis [...]. Notre époque hésite entre matérialisme et transcendance, pour faire de la mort une fin ou un commencement. Doit-on dans ce siècle désenchanté à la mémoire courte vaincre la mort par la science ou l'apprivoiser au travers d'un idéal réinventé?»

Enfin, à propos de gouvernance mondiale souhaitable (et quelques mois avant la conférence COP21 de Paris sur le changement climatique): «La génération actuelle est non seulement la première depuis longtemps à considérer que l'avenir de ses enfants sera moins radieux que le sien, mais à ne pas faire grand'chose pour éviter à ses descendants le lourd fardeau d'erreurs politiques, écologiques ou financières accumulées.» Le livre bénéficie d'une préface de Robert Badinter (ministre de la Justice de François Mitterrand, qui a fait abolir la peine de mort en 1981). Ceci bien que, dit Badinter, «nous n'appartenions pas à la même famille politique mais il s'agit de sujets qui transcendent les oppositions.» Quelques lignes: "Dans ce combat prométhéen [avec la mort], l'homme a fait reculer les barrières de l'inconnu et fait progresser sans cesse les savoirs et techniques. Mais, toujours infranchissable, la ligne qui demeure à l'horizon lui rappelle que demain il sera mort, c'est-à-dire autre ou rien.» «Je pense qu'il est temps que, dans les Déclarations des droits humains, soit inscrit le droit de ne pas souffrir, avec comme corollaire le droit aux soins palliatifs et à la sédation profonde.» 